\title{
DETERMINANTS OF HYPERGLYCEMIA IN VERY LOW BIRTH WEIGHT INFANTS
}

\section{Imene Ayadi Dahmane, Emira Ben Hamida, Mouadh Bou Ali, Bassem \\ Rabaii, Zahra Marrakchi \\ Department of Neonatology. Charles Nicolle Hospital. Tunis. Tunisia.}

\section{Introduction:}

Transient hyperglycemia is common in preterm infants. It is associated with increased risk of morbidity and mortality.

The aims of the study were to determine the prevalence of transient hyperglycemia in the extreme preterm infant, its risk factors, and its short-term outcomes.

\section{Methods :}

We conducted a retrospective study in the neonatal intensive care unit of Charles Nicolle Hospital of Tunis over a three and a half years period (January 2011-June 2014).

We included preterm infant

with low birth weight $(<1500 \mathrm{~g})$ and gestational age $<30$ weeks.

We defined two groups: "Hyperglycemic 》 and « Normoglycemic ».

We defined three classes of hyperglycemia: moderate $(1,8$ et $2,5 \mathrm{~g} / \mathrm{l})$, severe $(2,5-4,5 \mathrm{~g} / \mathrm{l})$ and uncontrolled $(>4,5 \mathrm{~g} / \mathrm{l})$.

We considered early hyperglycemia if occurring within the first 48 hours of life.

\section{Results:}

Prevalence of hyperglycemia was $36.8 \%$. Hyperglycemia was moderate, severe and uncontrolled in $15.4 \%, 66.6 \%$ and $18 \%$ of cases, respectively.

\begin{tabular}{|l|c|}
\hline \hline \multicolumn{2}{|c|}{ General characteristics of the "Hyperglycemia" } \\
group
\end{tabular}

\begin{tabular}{|c|c|c|c|}
\hline \multicolumn{4}{|c|}{ Risk factors for transiant hyperglycemia } \\
\hline & Hyperglycémie & Normoglycémie & $\mathbf{p}$ \\
\hline $\begin{array}{l}\text { Gestationnel } \\
\text { age }\end{array}$ & $\begin{array}{c}28,2 \pm 1,2 \\
{[25-30]}\end{array}$ & $\begin{array}{c}28,8 \pm 1,0 \\
{[26-30]}\end{array}$ & $<10^{-3}$ \\
\hline $\begin{array}{l}\text { Birth weight } \\
\text { (g) }\end{array}$ & $\begin{array}{c}1114,87 \pm \\
200 \\
{[780-1500]}\end{array}$ & $\begin{array}{c}1240,75 \pm \\
207[800- \\
1500]\end{array}$ & 0,003 \\
\hline IUGR (\%) & $23 \%$ & $17 \%$ & 0,002 \\
\hline CRIB score & $3[0-9]$ & $2[0-9]$ & $10^{-3}$ \\
\hline $\begin{array}{l}\text { Respiratory } \\
\text { distress } \\
\text { syndrome (\%) }\end{array}$ & 24,5 & 12,3 & 0,024 \\
\hline $\begin{array}{l}\text { Cumulative } \\
\text { carbohydrate } \\
\text { intake S1 } \\
\text { (g/kg) }\end{array}$ & $\begin{array}{l}99,9 \pm 14,8 \\
{[68,4-136,8]}\end{array}$ & $\begin{array}{l}92,0 \pm 14,0 \\
{[55,8-112,6]}\end{array}$ & 0,021 \\
\hline $\begin{array}{l}\text { Cumulative } \\
\text { caloric intakes } \\
\text { S1 (Kcal/kg) }\end{array}$ & $\begin{array}{l}536,9 \pm 127 \\
{[235,2-781,4]}\end{array}$ & $\begin{array}{l}643,8 \pm 151,3 \\
{[447,4-1098,8]}\end{array}$ & 0,002 \\
\hline $\begin{array}{l}\text { Cumulative } \\
\text { protidic } \\
\text { intake S2 } \\
(\mathbf{g} / \mathbf{k g})\end{array}$ & $\begin{array}{l}51,4 \pm 7,3 \\
{[40-71,7]}\end{array}$ & $\begin{array}{l}63,4 \pm 24,7 \\
{[35,6-131,9]}\end{array}$ & 0,029 \\
\hline $\begin{array}{l}\text { Cumulative } \\
\text { caloric intake } \\
\text { S2 (Kcal/kg) }\end{array}$ & $\begin{array}{l}1279,2 \pm 215,3 \\
{[871,9-1815,8]}\end{array}$ & $\begin{array}{c}1532,7 \pm 316,2 \\
{[1028,7-2307} \\
6]\end{array}$ & $<10^{-3}$ \\
\hline
\end{tabular}

Conclusion: We found a high prevalence of hyperglycemia in the preterm infant. A preventive strategy of accessible risk factors is necessary to lower the prevalence of this metabolic disorder responsible of important morbidity and mortality. 